\title{
Cross-Training Policies for Team Cost and Robustness
}

\begin{abstract}
We assess alternative cross-training policies for work-teams considering cost, and levels of cross training. The policies are assessed with respect to their robustness to demand-mix variation and absenteeism coverage. We employ simulation to examine instances where cross training can be used to help meet a fixed demand scenario, and with instances where cross-training can help to meet demand mix variability. Current results indicate that when minimizing cross-training costs, policies related to equalizing the cross-training level among the workforce, may provide improvement in terms of robustness without additional cost. We also assess the effects of some environmental factors, demand mix-coverage, absenteeism coverage, and job-task correlation.
\end{abstract}

\section{Introduction}

There are several competing objectives in the problem of improving team cross-training, including job enrichment, worker health and safety, motivating the workforce, and flexibility to deal with absent workers (Bokhorst and Slomp, 2007). However, training costs, learning, cognitive loads, scheduling, and demand, all create challenges and complexity to the decision making process. Determining an appropriate cross-training level for an organization should ideally consider some of these issues. In this paper, we focus our efforts on identifying low cost cross-training policies and the robustness of those policies with respect meeting levels of Demand-Mix Coverage (DMC) and Absenteeism Coverage (AC). We will address instances where cross-training is intended to meet Fixed Demand (FD), AC, and DMC.

The literature has considered the problem of obtaining appropriate cross-training levels under certainty. Brusco and Johns (1998) considered several potential cross-training policies in the maintenance service of a paper mill factory, concluding that a skill chaining policy has the ability to aid in workload balancing. Jordan et al. (2004) conducted several experiments to show that complete chains (i.e., chains that taken together create worker redundancy for all tasks), are critically important, and without which performance may be significantly degraded. 
Agnihothri and Mishra (2004) examined an equipment repair service, wherein travel time and downtime cost were considered. Historical data were used to forecast future requirements, where they conclude that full cross-training is sometimes the most cost effective policy in field service operations. Chakravarthy and Agnihothri (2005) modeled the mix between specialist and generalist workers in a service system with two clients and stochastic demand. Also, Wirojanagud et al. (2007) considered a semiconductor manufacturing industry in which all workers must be cross-trained. The consideration of demand coverage has also been considered in specific domains including computer science (e.g., Giannikos 2010), transportation (e.g., Fei and Mahmassani 2011) and hospitals (Gnanlet and Gilland, 2014).

It is unusual for work units to have known, constant work requirements over anything more than trivially short time horizons. More often demand is uncertain and work teams must be flexible to adapt to this variation. Cross-training can be used to cope with variable requirements. In general, more cross-training implies greater flexibility, which is in turn necessary to cope with demand variability (Easton, 2011). But cross-training usually have a cost and because of it has to be cautiously implemented. Consequently, the literature has presented several cross-training policies to moderate the relationship between cross-training levels and demand variability.

With this aim, policies that provide significant outcome flexibility with a limited flexibility of resources have been reported. Chaining strategies, applied to production lines, provide outcome flexibility not far from the maximum, with limited cross-training levels (Brusco and Johns 1998). A $D$-skill chaining pattern assigns workers to $D$ adjacent stations. The broader benefits of skill chaining in serial production lines has also been analyzed by Hopp et al. (2004). Gong et al. (2011) studied a U-shaped line, staffed with skill-chained workers with absenteeism, and proposed a policy for cross-training, wherein workloads are balanced by only crosstraining them on certain stations. Overall, with modest capacity imbalance and significant variability, the strategies based on skill chaining are particularly robust.

In the other side, advantages have also been found in dedicated-specialized schemas, in which some workers are cross-trained on few to no tasks, and others are highly cross-trained. The so-called cherry-picking strategy assigns workers with excess resource capacity to other workstations to improve line balance with a minimum amount of cross-training (Tekin et al., 
2002). In the current study, we similarly consider absenteeism in the context of cross-training policies, and examine several policies related to cherry-picking approaches, for systems with demand-mix requirements that must be fulfilled.

We assume that when variability of demand and absences are not taken into account, crosstraining policies based on minimum levels tend to be overestimated, as the benefits of additional capacity are not included. In this line of work, Easton (2014) showed that when ignoring the joint variability of attendance and demand, workforce scheduling models tend to overstate the benefits of cross-training. The current work considers alternative team crosstraining policies considering cost and levels of cross training. The policies are assessed with respect to their robustness to demand-mix variation and absenteeism exceeds the forecasts. We posit that this approach corresponds to real needs in defining cross-training and, has not been previously dealt with in the literature.

As mentioned previously, the effect of cross-training on demand coverture can depend on context. To test the dependence on the context of the particular effects analyzed here, two environmental factors affecting the relation between cross-training and demand coverture have been defined and considered. The first factor measures the similarity between the tasks to be performed to complete the different jobs. The second factor takes into account the level of demand variability that have to be coped with.

In the remainder of this paper, we present our methodology for policy evaluation in Section 2 , followed by the corresponding results and discussion in Section 3. Section 4 concludes the paper with overall findings, limitations, and future directions.

\section{Methodology}

The objective of this paper is to assess alternative cross-training policies for a work-team. Cost, equal cross-training, specialized workers and different combinations of these policies are considered in scenarios where we use cross-training to first deal with fixed demand, FD and absenteeism coverage, AC, and then secondly to jointly consider demand-mix-coverage, DMC and absenteeism coverage. 
We note that a full cross-training scenario, in which all workers cross-trained on all tasks is relatively rare in practice, particularly for large work centers, though it may be practical for smaller work teams or organizations with more limited task sets. There are certainly work teams for which full cross-training is unreasonable, including high knowledge intensity settings such as airline crews, or surgical teams. Nonetheless, we consider the potential for full crosstraining. But cases where initial qualification and position allows workers to perform any of the tasks assigned to the team are also numerous, both in manufacturing and service settings. We will also assume that no particular resource constraints will apply, and consequently, the only condition to cover certain demand is that the workers have time enough to complete the tasks

We consider five cross-training policies to include:

(1) Minimize training cost - the total number of tasks for which workers are trained, with an assumed constant cost across tasks;

(2) Maximize number of specialists, where specialists are specific workers who are trained in no more than a certain number of tasks, and secondarily minimizing the cross-training cost.

(3) Minimize cross-training level, assuming an equal level for all workers.

Alternatively, maintaining a minimum cross-training cost can be established as constraint, which in turn creates two additional multi-level policies, including,

(4) Minimize the training cost and then maximize the number of specialists;

(5) Minimize training cost and then minimize the cross-training level.

Thus, policy (4) places preemptive priority on policy (1), then (2). Policy (5) places preemptive priority on (1), then (3).

We consider a set of products and a corresponding set of tasks required for these products, and simulate instances with fixed demand as well as instances where a set of demand-mix coverage scenarios must be met. We are initially examining whether cross-training is robust enough to address levels of demand-mix variability alongside an uncertain level of absenteeism. We include at least a base level of absenteeism in all instances, given its ubiquity in practice. 
We establish levels of DMC by setting limits on the proportion of time devoted to each product. For example, in a three-product scenario, a team could be required to meet all demands, given that no more that $60 \%$ of work time went to product 1 , no more than $50 \%$ to product 2 , and no more than $70 \%$ to product 3 . Thus, a $25 \%, 25 \%$ and $50 \%$ split of work among products 1, 2 and 3, respectively, would be acceptable, while an $80 \%, 10 \%$ and $10 \%$ split would not. This approach was proposed by Olivella and Nembhard (2015).

Two environmental variables are taken into account. First, Job-Task Correlation (JTC) is a measure of the similarities among jobs within the demand-mix, based on completion times of the lower level tasks that comprise the jobs. This is ultimately based on organizational level decisions, and strongly conditioned on the characteristics and design of the work. The second environmental variable is the level of DMC to be met.

We will assess the application of the strategies by considering three resultant outcome measures: cross-training cost, robustness with respect to demand-mix coverage, and robustness given workers' absences. We define the robustness with DMC as the capacity to deal with demands that exceed the bounds of the DMC that are determined a priori. As cross-training is defined to cover the DMC between the bounds, with demands that exceed the bounds, some work will remain undone. Thus robustness relates to the amount of unmet demand that exceeds the DMC bounds. Similarly, robustness regarding worker absence is related to the amount of unmet demand caused by absenteeism.

We consider two models, first a model to obtain a cross-training solution that allows a team to meet a DMC level with a given AC level, under alternative objective functions. This model will also be used in instances with fixed demand, FD. The second model is intended to obtain the amount of unmet demand when a team is cross-trained to meet DMC and AC levels. In addition, an experimental design is defined. The detail are presented next.

\section{Model to determine cross-training}

Data

A work team is formed by a set of workers that are able to perform any of a set of tasks if they are expressly trained for it. A set of products are produced in variable amounts, according to 
demand (henceforth demand scenarios) that are convolutions of quantities of planning blocks, formed by a number of product units.

J Number of different products $(j=1 . . J)$.

$K \quad$ Number of different kinds of tasks necessary to obtain the products $(k=1 . . K)$.

$W \quad$ Number of workers $(w=1 . . W)$.

A Upper bound on the number of absent workers.

H

Total work time per worker during the planning period in a given time unit -the examples have been thought in hours.

$v_{k j} \quad$ Number of units of time necessary to perform task $k$ in order to complete a planning block of product $j$.

$m_{j} \quad$ Parameters for the level of demand coverage (\%) that the team has to meet for product $j$. This is an upper bound on the proportion of time that that is available for product $j$ considering cross-training and is proportional to DMC (i.e., workers must devote a proportion of the total available time to product $j$ for a range of demand scenarios from 0 to $\left.m_{j}, j=1 . . J\right)$. When a FD is considered these parameters sum to 1 .

$D \quad$ Number of demand scenarios that workers must meet with $(\delta=1 . . D)$. The demand scenarios account for demands and bounds with respect to time allocated to each product. We exclude trivially differential scenarios in order to maintain a manageable set. We note that this is without loss of generality since the set that results from adding one unit to some component will be part of other groups and yield the same or greater requirements.

$d_{\delta j} \quad$ For demand scenario $\delta$, the number of planning blocks of product $j(\delta=1 . . D, j=1 .$.$) .$

$P \quad$ Number of sets of present (non absent) workers $(\rho=1 . . P)$. These are generated taking into account the possible variations in the group of present workers when $A$ workers are absent. Sets with less than $A$ absents are not considered as they cannot 
determine more demanding requirements that sets with $A$ absences.

$p_{\rho w^{\prime}} \quad$ For the set of present workers $\rho$, worker $w^{\prime}\left(\rho=1 . . P, w^{\prime}=1 . . W-A\right)$.

$s^{\prime} \quad$ Number of tasks for which a worker can be cross-trained to be considered specialized.

$c_{k} \quad$ Cost of training a worker on task $k(w=1 . . W)$.

To produce a product unit, task times assigned to the workers are knows as well as each worker's work time during the planning period Workers are able to fulfill any demand scenario with some proportion of the total work time devoted to each product, and this is bounded above by a percentage.

The workers must perform, in spite of a level of absences, any demand scenario respecting the maximum level of DMC. The number of tasks on which each worker is to be cross-trained can have a lower bound, upper bound or be unbounded, according to the adopted crosstraining structure. The same condition can apply for all team members or, alternatively, the team can be divided into two subsets, one set formed by workers to be highly cross-trained and a second formed by workers to be minimally cross-trained. That is, we can classify workers as 'generalists' and 'specialists', respectively.

\section{Variable Definitions}

The objective is to obtain cross-training that allows a team to cope with demand scenarios fulfilling established bounds on demand mix coverage, DMC, despite a certain number of absences. Upper and lower bounds on the number of tasks per workers are maintained and the cost of cross-training is minimized. We formulate a math programming model below with the following additional notation.

$x_{w k} \quad$ Binary variable that indicates whether the worker $w$ is able to perform the task $k$ $(w=1 . . J, k=1 . . K)$

$y_{w k \rho \delta} \quad$ Number of units of time that worker $w$ devotes to the task $k$ in the assignment of work and given that the set of present workers $\rho$ is able to meet the demand 
scenario $\delta(w=1 . . W, k=1 . . K, \rho=1 . . P, \delta=1 . . D)$

$S_{w} \quad$ Binary variable that indicates whether the worker $w$ is specialized (i.e., trained on $s^{\prime}$ tasks or less).

$l_{1}, l_{2} \quad$ Smallest and biggest number of tasks in which the workers are cross-trained.

Constraints (1) to (3) are included so that each group of present workers is able to perform each possible demand scenario $\delta$. Constraint (1) imposes a work time bound for each worker, (2) implies that no work is assigned that is not part of the group of present workers, and (3) imposes that a solution to meet demand is obtained. Constraint (4) expresses that a worker devotes time only to tasks that $\mathrm{s} /$ he is able to perform and constraint (5) imposes that $S_{w}$ is 1 , that is to say, the worker is not specialized, when he is trained between s' +1 and an unlimited number of tasks $-S_{w}$ is the total number of tasks. Finally, constrain (6) is included only when the objective functions 3 or 5, defined below, are considered. Constrain (6), together with these objectives functions, make $l_{1}$ and $l_{2}$ the smallest and the biggest number of tasks, respectively, in which the workers are trained.

\begin{tabular}{|c|c|}
\hline$\sum_{k=1}^{K} y_{w k \rho \delta} \leq H, \quad w=1 . . W, \rho=1 . . P, \delta=1 . . D$ & $(1)$ \\
\hline$y_{w k \rho \delta}=0, \quad w=1 . . W, k=1 . . K, \rho=1 . . P, \delta=1 . . D, w \notin\left(p_{\rho 1}, . ., p_{\rho W-A}\right)$ & $(2)$ \\
\hline$\sum_{w=1}^{W} y_{w k \rho \delta} \geq \sum_{j=1}^{J} v_{k j} \cdot d_{\delta j}, \quad k=1 . . K, \rho=1 . . P, \delta=1 . . D$ & (3) \\
\hline$y_{w k \rho \delta} \leq x_{w k} \cdot H, \quad w=1 . . W, k=1 . . K, \rho=1 . . P, \delta=1 . . D$ & $(4)$ \\
\hline$\sum_{k=1}^{K} x_{w k} \leq s^{\prime}+S_{w} \cdot K, \quad w=1 . . W$ & (5) \\
\hline$l_{1} \leq \sum_{k=1}^{K} x_{w k} \leq l_{2}, \quad w=1 . . W$ & (6) \\
\hline
\end{tabular}




\section{Objective functions (Policies)}

The objective function determines the cross-training policy and thus is defined independently for each of the alternative policies considered. We now define the objective functions for each of the cross-training policies.

(1) Minimum cost policy. The objective function (7) considers only the objective of minimizing the cross-training cost, obtained by adding the number of workers to be trained in each task by the cost of training a worker in this task.

$$
\min \sum_{w=1}^{W} \sum_{k=1}^{K} x_{w k} \cdot c_{k}
$$

(2) Maximum number of specialized workers policy. The objective consists on maximizing the number of specialized workers and secondarily minimizing the cross-training cost. The second term of the function is the cross-training cost divided by a quantity that make it less than 1 in order that the objective of minimizing the cost of cross-training was secondary.

$$
\min \sum_{w=1}^{W} S_{w}+\frac{\sum_{w=1}^{W} \sum_{k=1}^{K} x_{w k} \cdot c_{k}}{W \cdot \sum_{k=1}^{K} c_{k}+1}
$$

(3) Equal cross-training policy. The objective is minimizing the cross-training cost with all workers cross-trained in the same number of tasks.

$$
\min l_{2}-l_{1}+\frac{\sum_{w=1}^{W} \sum_{k=1}^{K} x_{w k} \cdot c_{k}}{W \cdot \sum_{k=1}^{K} c_{k}+1}
$$

(4) Minimum cost and specialized workers policy. The objective consists on minimizing the cross-training cost and secondarily maximizing the number of specialized workers. The second term of the equation is the number of specialized workers multiplied by the minimum cost and divided by the number of workers plus 1 , which guarantee that the second term is always 
minor that the smallest component of the summation of the first term and, in consequence, that the second term is a secondary objective.

$$
\min \sum_{w=1}^{W} \sum_{k=1}^{K} x_{w k} \cdot c_{k}+\sum_{w=1}^{W} S_{w} \cdot \frac{\min \left(c_{1}, . ., c_{K}\right)}{W+1}
$$

(5) Minimum cost and equal cross-training policy. The objective is minimizing the crosstraining cost and secondarily minimizing the higher number of tasks in which a worker is crosstrained. The second term of equation is a secondary objective because of the same reasons that in the objective function (4).

$$
\min \sum_{w=1}^{W} \sum_{k=1}^{K} x_{w k} \cdot c_{k}+\left(l_{2}-l_{1}\right) \cdot \frac{\min \left(c_{1}, . ., c_{K}\right)}{W+1}
$$

\section{Model to Quantify Unmet Demand}

Since we are also interested in assessing the ability of cross trained teams to deal with situations in which the demand-mix or absenteeism is significantly elevated, we consider bounds on cross training to inform the quantification of its utility. When FD is considered, the effect of a change of the demand mix is also considered, as can happen in practice even when the demand is initially fixed.

Given a level of cross-training, a demand scenario and set of present workers, we can determine the level of unmet demand. We define a math programming model for assigning the workers to tasks by considering cross-training and minimizing the time necessary to complete the unmet demand. The model includes constants $J, K, W, A, H$, defined above, and the following.

Data

$x^{\prime}{ }_{w k} \quad$ For the work team cross-training considered, binary variable that indicates whether the worker $w$ is able to perform task $k(w=1 . . J, k=1 . . K)$

$d_{j}^{\prime} \quad$ For the demand scenario considered, the number of planning blocks of the product 


$$
j(\delta=1 . . D, j=1 . . J) \text {. }
$$

$p^{\prime} w^{\prime} \quad$ For the set of present workers considered, worker $w^{\prime}\left(w^{\prime}=1 . . W-A\right)$.

\section{Variables}

$y^{\prime}{ }_{w k} \quad$ Number of units of time that the worker $w$ would devote to the task $k(w=1 . . W$, $k=1 . . K)$

$u_{k} \quad$ Positive difference between the units of time necessary to meet the demand and the total work time of the workers on that task $k(k=1 . . K)$. We note that a positive difference is because the demand scenario cannot be covered, and a negative difference indicates the completion of unnecessary work.

Constraint (12) imposes the work time bound for each worker; (13) implies that no work is assigned to an worker that is not part of the group of present workers; (14) imposes that the work done plus the positive difference between the units of time necessary to meet demand and the units of time performed by the workers is equal to the units of time necessary to meet demand, for each kind of task; constraint (15) expresses that a worker devotes time only to tasks that $\mathrm{s} /$ he is able to perform, and constraint (16) imposes bounds on the number of tasks for which a worker can be trained.

$$
\begin{aligned}
& \sum_{k=1}^{K} y_{w k}^{\prime} \leq H, \quad w=1 . . W \\
& y^{\prime}{ }_{w k}=0, \quad w=1 . . W, k=1 . . K, w \notin\left(p_{1}, . ., p_{W-A}\right) \\
& \sum_{w=1}^{W} y^{\prime}{ }_{w k}+u_{k} \geq \sum_{j=1}^{J} v_{k j} \cdot d^{\prime}{ }_{j}, \quad k=1 . . K \\
& y^{\prime}{ }_{w k} \leq x^{\prime}{ }_{w k} \cdot H, \quad w=1 . . W, k=1 . . K
\end{aligned}
$$

\section{Objective function}


The objective function consists of minimizing unmet demand, measured by taking the difference between the units of time necessary to meet the demand and the number of time units of work corresponding to the work that the workers are able to perform (16).

$$
\sum_{k=1}^{K} u_{k}
$$

\section{Experimental Design}

We conduct an experiment including 4,000 randomly generated instances. Within this set, 2,000 are instances where cross-training is used to meet a fixed demand, while in the other 2,000 instances cross-training is used to meet scenarios with demand-mix variation. In all cases, absences are included to represent common practice. The experimental design includes three experimental factors including, one policy factor, and two environmental factors (job-task correlation, and level of demand mix coverage). We also measure three response variables (cross training cost, unmet demand due to DMC, and unmet demand due to AC).

Even some methodologies to predict individual absenteeism are developed in the literature, a model to be applied to a general case is not available, until the authors know. At the same time, the variability of the demand-mix that exceed the forecast seems difficult to estimate. Because of this, the factors and scenarios have been defined to reflect a wide range of possible variations. Experiment factors and factors levels are defined in Table 1. Details about how the scenarios were generated are given in Table 2.

Table 1. Experimental Design

\begin{tabular}{lll}
\hline \multicolumn{2}{c}{ Factor / Variable } & \multicolumn{1}{c}{ Measure } \\
\hline Policy Factor & Cross-training strategy & $\begin{array}{l}\text { Five levels: minimum cost; specialized workers; equal } \\
\text { cross-training; minimum cost and specialized workers; } \\
\text { and minimum cost and equal cross-training }\end{array}$ \\
$\begin{array}{l}\text { Environmental Job-Task correlation (JTC) } \\
\text { Factors }\end{array}$ & $\begin{array}{l}\text { Average correlation of task requirements across jobs, and } \\
\text { reflects similarities among jobs within the demand-mix }\end{array}$ \\
& $\begin{array}{l}\text { Demand-Mix Coverage level } \\
\text { (DMC level) }\end{array}$ & $\begin{array}{l}\text { An upper bound on the proportion of time that can be } \\
\text { devoted to each product -it is taken into account in the } \\
\text { instances with demand-mix variability. }\end{array}$
\end{tabular}




\section{Response Cross-training cost Variables}

Unmet demand when demand-mix coverage bounds are exceeded

Unmet demand when absenteeism bound is exceeded
The cost of the minimum cross-training needed to verify the established conditions

Average of the unmet demand by considering a set of instances with demand-mix coverage exceeding bounds. Even when FD is considered, variations are possible

Average of the unmet demand considering a set of instances with absenteeism exceeding established bounds

\section{Variation of Cross-training strategies}

Cases

Bounds on the demand-mix coverage to be met -it is taken into account in the instances with demand-mix variability.

Job-task structure: to complete each job, which tasks have to be performed and time to devote to each task

\section{Table 2. Experimental Details}

Scenario $\quad \operatorname{Products}(J)=4 ; \operatorname{Tasks}(I)=5$; $\operatorname{Workers}(W)=10 ; \operatorname{Absentees}(A)=1 ; c_{1}, . ., c_{W}=1 ; s^{\prime}=1$

Instances Time of task $i$ (columns) to complete a unit of product $j$ (rows) $\left(v_{k j}\right)$ : The instances the randomly generated in two steps. In the first step it is randomly established for each component whether it will be 0 or a non-null number, with probability 0.5 for both possibilities. When a non-null number has to be assigned, a number between 0 and 1 is randomly chosen. If all the values corresponding to a product are 0 , the calculation is repeated for this product. A total of 400 instances for with FD and 20 instances with DMC.

Upper bounds on proportion of time devoted to products $1 . . J\left(p_{\mathrm{j}}\right)$ : The instances are randomly generated by assigning each component a randomly generated value between .05 and 1 . A total of 20 instances for the cases with demand-mix variation are generated.

Cross-trained policies: A, minimum cost; $\mathrm{B}$, maximum number of specialized workers; $\mathrm{C}$, equal cross-training; D, policy A and secondarily policy B; and E, policy A and secondarily policy $\mathrm{C}$.

For each Unmet demand when DMC is exceeded: For each of the demand scenarios that the Instance workers must meet $(\delta=1 . . \Delta)$, we consider the demands that result from adding 1 to each product, respectively, and we calculate the average of the unmet demands obtained. 
Unmet demand when the absenteeism bound is exceeded: All the combinations with 2 absent workers are considered, and we calculate the average of the unmet demands obtained.

Parameters Planning blocks: three or more planning blocks per product are taken, which are less than or equal to $10 \%$ of total work time, assuming that the goal is to define as few blocks as possible

$H=160$

Number of scenarios to select for each calculation attempt: 10

\section{Results and Discussion}

The experiment has been performed using IBM ILOG CPLEX 12.1.0 on an Intel(R) Pentium(R) D 3.4GHz with 2GB RAM. In all cases exact solutions were obtained. We summarize the results of the experiments below based on ANOVA and formal contrasts. We begin with the most general results of the experiment with respect to the three factors and their relative importance and significance. We then discuss details corresponding to each of the individual factors.

Table 3 presents a summary of the results from the ANOVA for each of the three response variables considered (Cost, unmet demand with DMC, and unmet demand with absenteeism). The table also combines results from considering both a fixed demand, FD, and Demand-mix case. The unmet demand variables will allow quantifying the robustness of the policies, in addition to considering the overall policy costs.

For each of the variables and both for FD and DMC, the hypotheses of the influence of the cross-training policy on the demand-mix coverage, $D M C$, level is supported. The job-task correlation, JTC, is evidenced to be significant although the results are not as strong.

Table 3. ANOVA Summary

\begin{tabular}{|c|c|c|c|c|c|}
\hline \multirow[b]{2}{*}{ Source } & \multirow[b]{2}{*}{ Variable } & \multicolumn{2}{|c|}{ Fixed demand $^{\mathbf{1}}$} & \multicolumn{2}{|c|}{$\begin{array}{c}\text { Demand-mix } \\
\text { Coverage }^{2}\end{array}$} \\
\hline & & $\boldsymbol{F}$ & Prob. $>F$ & $\boldsymbol{F}$ & Prob. $>F$ \\
\hline Policy & Cross-training cost & 1845.29 & $<0.001$ & 169.02 & $<0.001$ \\
\hline
\end{tabular}




\begin{tabular}{|c|c|c|c|c|c|}
\hline Policy & Unmet Demand when DMC bounds are exceeded & 290,74 & $<0.001$ & 18.37 & $<0.001$ \\
\hline Policy & Unmet Demand when AC bound is exceeded & 335,95 & $<0.001$ & 30.83 & $<0.001$ \\
\hline JTC & Cross-training cost & 10,71 & $<0.001$ & 16.14 & $<0.001$ \\
\hline JTC & Unmet Demand when DMC bounds are exceeded & 44,79 & $<0.001$ & 3.31 & $<0.001$ \\
\hline JTC & Unmet Demand when AC bound is exceeded & 6,93 & $<0.001$ & 2.29 & 0.001 \\
\hline DMC Level & Cross-training cost & & & 89.37 & $<0.001$ \\
\hline DMC Level & Unmet Demand when DMC bounds are exceeded & & & 251.06 & $<0.001$ \\
\hline DMC Level & Unmet Demand when AC bound is exceeded & & & 180.25 & $<0.001$ \\
\hline
\end{tabular}




\section{Results by Policy}

Given the significance of each of the three factors including the cross training policy factor, we next contrast the five levels of this factor. Table 4 summarizes the average, standard deviation of cost, unmet demand due to mix, and unmet demand due to absences, by policy. Confidence Intervals (CI) at 95\% are given of the results and are illustrated in Figure 1-3.

Table 4. Results of the Response Variables per Policy

\begin{tabular}{ccccccccccccccc}
\hline & \multicolumn{1}{c}{} & \multicolumn{1}{c}{ With Fixed Demand } & & \multicolumn{1}{c}{ With Demand-Mix Coverage } \\
\hline & & & Cost & & $\begin{array}{c}\text { Unmet Demand } \\
\text { Due to Mix }\end{array}$ & $\begin{array}{c}\text { Unmet Demand } \\
\text { Due to Absences }\end{array}$ & Cost & $\begin{array}{c}\text { Unmet Demand } \\
\text { Due to Mix }\end{array}$ & $\begin{array}{c}\text { Unmet Demand } \\
\text { Due to Absences }\end{array}$ \\
\hline Policy & N & Mean & StDev & Mean & StDev & Mean & StDev & Mean & StDev & Mean & StDev & Mean StDev \\
\hline A & 400 & 16.07 & 0.91 & 67.50 & 32.72 & 35.87 & 15.5 & 22.18 & 3,34 & 10.57 & 20.76 & 7.08 & 11.11 \\
\hline B & 400 & 16.07 & 0.91 & 88.45 & 32.25 & 47.47 & 16.02 & 22.20 & 3,38 & 14.26 & 25.89 & 9.59 & 13.80 \\
\hline C & 400 & 20.00 & 0.00 & 26.02 & 21.97 & 14.48 & 11.35 & 27.45 & 4,53 & 3.31 & 9.1 & 2.05 & 3.83 \\
\hline D & 400 & 16.07 & 0.91 & 87.18 & 31.79 & 47.11 & 15.47 & 22.18 & 3,34 & 13.89 & 25.3 & 9.32 & 13.27 \\
\hline E & 400 & 16.07 & 0.91 & 58.64 & 29.65 & 31.92 & 14.91 & 22.18 & 3,34 & 9.16 & 17.87 & 6.53 & 9.60 \\
\hline
\end{tabular}

A. Minimum cost; B. Maximum number of specialized workers; C. Equal cross-training; D. Policy A and secondarily policy B: E. Policy A and secondarily policy C

The summary of the cross-training cost by policy is presented in Figure 1, wherein the minimum cost policy, $A$, naturally led to the lowest cost overall. With a minimum cost policy followed by a secondary priority policy, (i.e., policies $D$ and $E$ ) no further cost reduction was obtained. We note that, while these secondary priorities yield a trivial result for the crosstraining cost measure, we will later compare these with respect to the unmet demand variables, wherein we will note performance differences among the three policies.

The policy of maximizing the number of specialized workers, $B$, only slightly increases the cost over the minimum cost policy in the case with $\mathrm{DMC}$, and maintains this cost when covering FD. That is, by maximizing the number of specialized workers, the cross-training cost itself is almost minimized. By contrast, the policy of equal cross-training, $C$, significantly 
increases the cost. This is possibly due to the notion that in matching cross-trained workers it may be necessary to cross-train more of them, even if it is not necessary in order to meet demand.

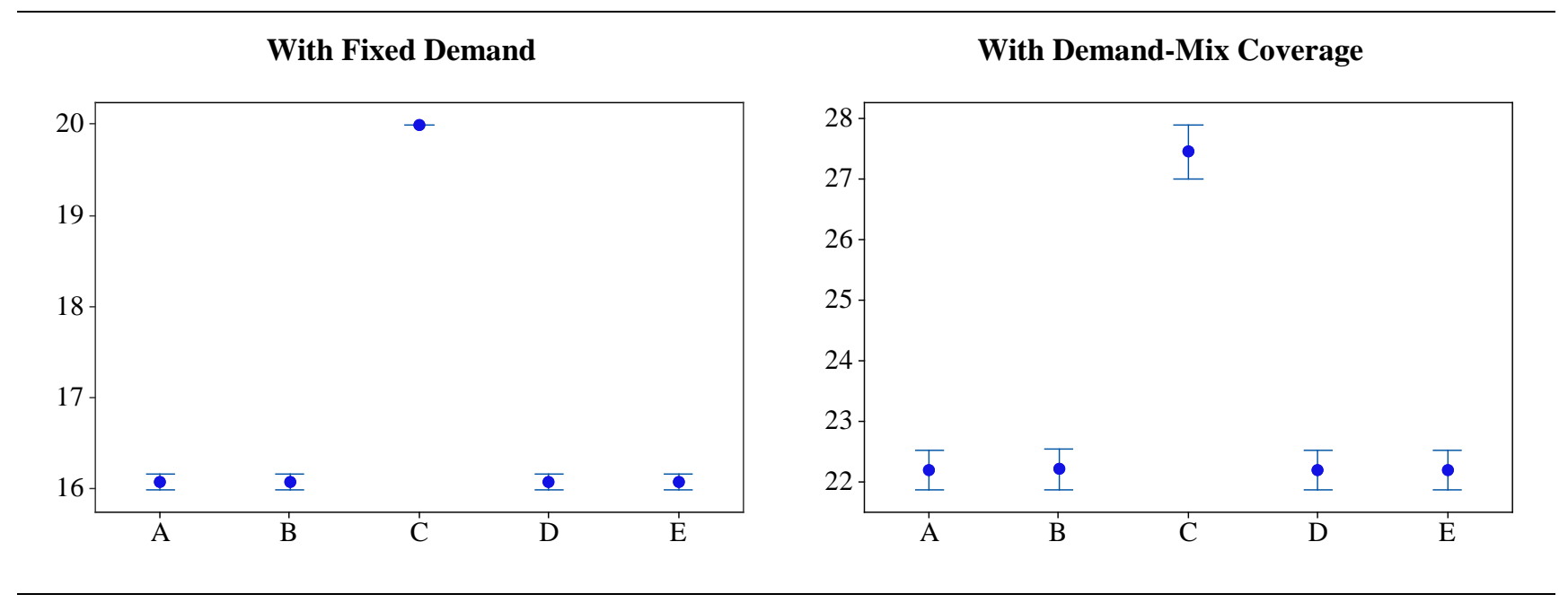

A. Minimum cost; B. Maximum number of specialized workers; C. Equal cross-training; D. Policy A and secondarily policy B: E. Policy A and secondarily policy C

\section{Figure 1. CI of Cost by Cross-Training Policy}

In Figure 2, the CI presents the unmet demand when the bounds on DMC or absenteeism are exceeded. Policy C, (equal cross-training), yields the least unmet demand relative to the other policies, both with FD and with DMC. However, from Figure 1, we also know that this policy is associated with higher costs. This is similar to earlier results that conclude that policies that don't specifically minimize cross-training tend to involve elevated costs when compared to specialization (e.g., Easton 2011, Nembhard and Bentefouet 2012). More interestingly, with policy E, which gives first priority to cross-training cost and secondarily maximizes the equity of cross-training across workers, the unmet demand is less than when only minimizing crosstraining cost (policy A). Thus, policy E increases the capacity to deal with cases that exceed DMC bounds without any additional cost. Thus, in some settings policy E may be more robust than policy A. Conversely, policy B (maximize the number of specialized workers), led to marginally higher levels of unmet demand when DMC bounds were exceeded, when applied as a primary or as a secondary criterion. Similar conclusions may be drawn from the results from both fixed demand and demand-mix scenarios. 


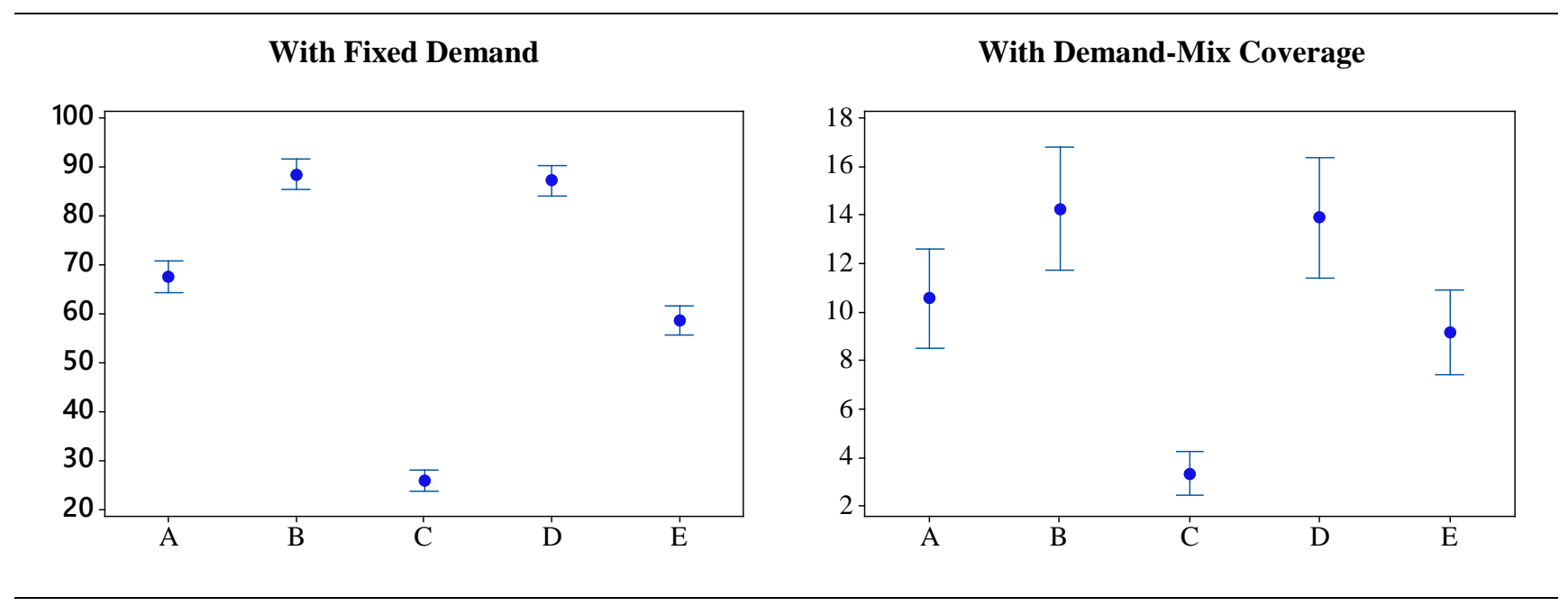

A. Minimum cost; B. Maximum number of specialized workers; C. Equal cross-training; D. Policy A and secondarily policy B: E. Policy A and secondarily policy C

\section{Figure 2. CI of the Unmet Demand Due to Demand-Mix per Policy}

Figure 3 illustrates the results considering absenteeism. We firstly observe that the results are similar to those for demand-mix and there is a clear tradeoff between minimizing costs, and minimizing unmet demands. Policy B (max specialization) significantly elevate cost over policy A, as seen before, and at the same time significantly elevate levels of unmet demand due to absenteeism, as it does with unmet demand due to demand-mix. The equal cross-training policy reduces unmet demand, even when applied as a secondary criterion. The results are similar with FD and with DMC. Thus, a secondary priority (e.g., policy E) can increase the robustness of the straightforward minimize cost policy (A), both against demand-mix variability and absenteeism. This may provide managers potentially valuable policy alternatives. 


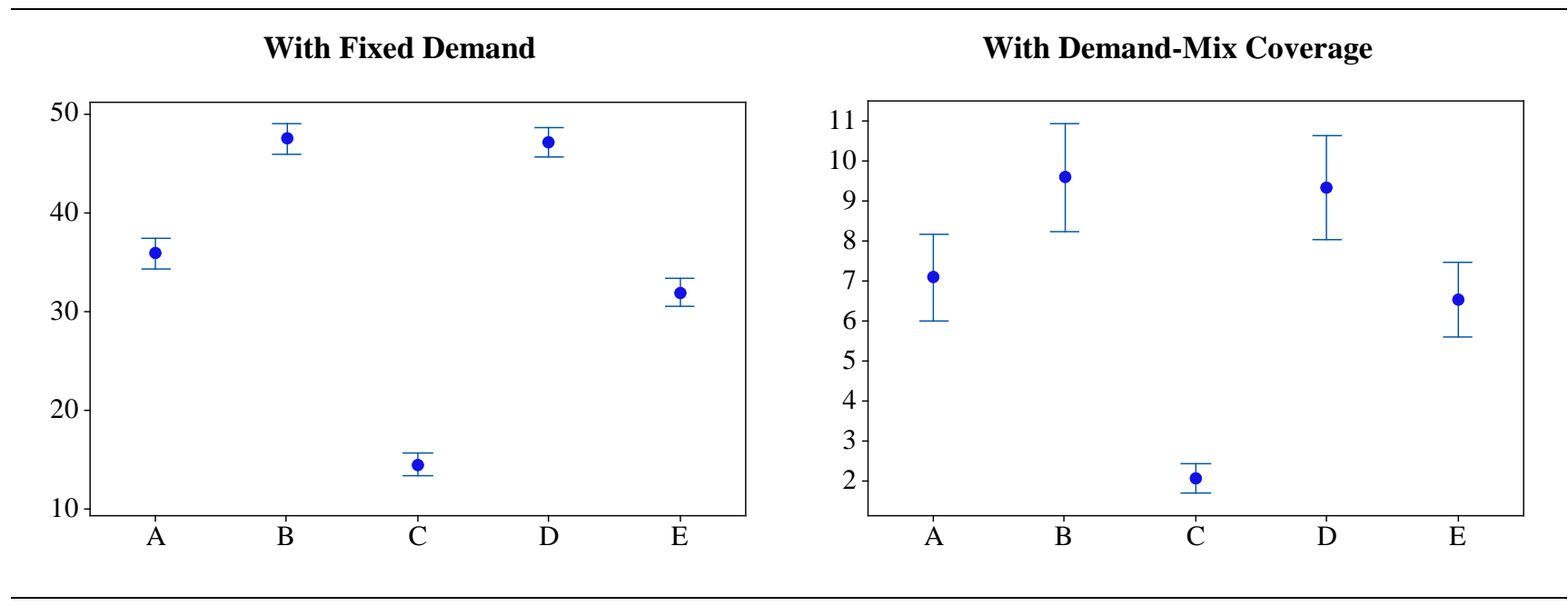

A. Minimum cost; B. Maximum number of specialized workers; C. Equal cross-training; D. Policy A and secondarily policy B: E. Policy A and secondarily policy C

\section{Figure 3. CI of the Unmet Demand Due to Absences per Policy}

Regression analysis of the influence of the environmental variables

We examine the influence of the two environmental factors on the three response variables using regression models. First, the influence on the cross-training cost is addressed. The relationships of the environmental factors with the cross-training cost are shown in Table 5, wherein the JTC is negatively related with cross-training cost with DMC ( $t$-test, $p<0.001)$ and no significant result in the fixed demand case. The negative relation with DMC suggests that on average, the greater the extent of job similarity, the less cross-training is necessary. This aligns well with suggestions made in some previous studies (e.g., Marentette 2009). In addition, in the results of the instances with $\mathrm{DMC}$, DMC level is positively related with the cross-training cost, and by consequence, when DMC is higher, a greater level of cross-training is necessary to meet demand.. 
Table 5. Regression analysis of cross-training cost in relation to environmental variables

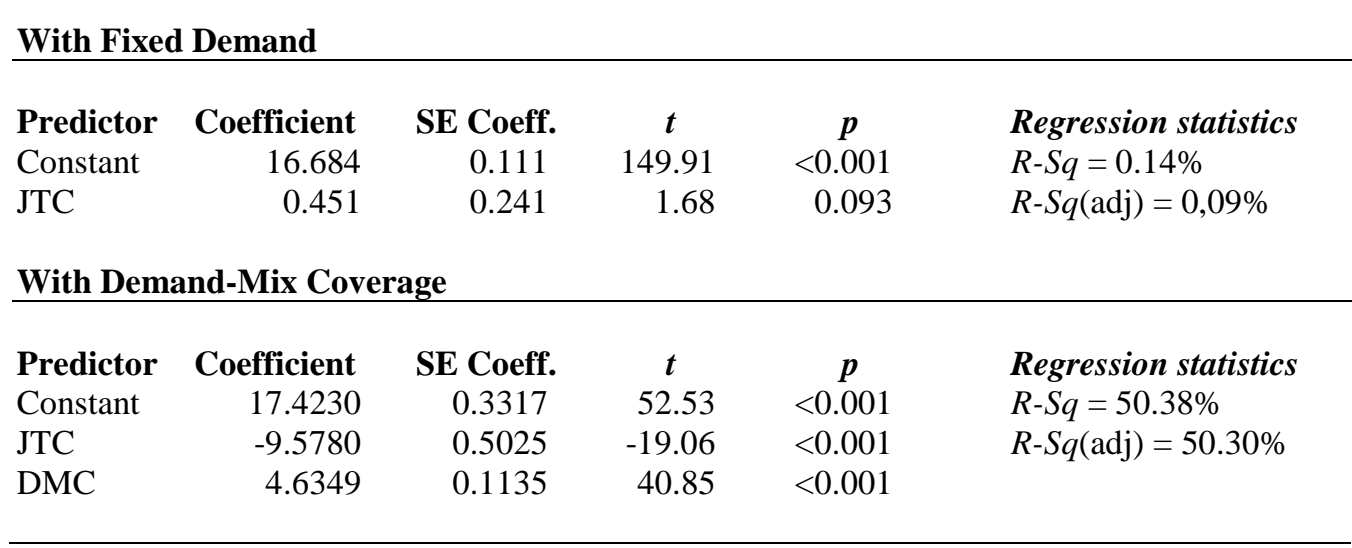

The influence of the environmental variables on situations in which DMC results in unmet demand yields results as shown in Table 6. Similarly, Table 8 summarizes the findings for umet demand resulting from Absenteeism. From Table 6, we note that JTC is negatively related with unmet demand when bounds are exceeded both with FD and with DMC. This naturally suggests that as DMC increases, the level of unmet demand will decrease. We remark that other research has shown some advantages of variation for human paced production systems (e.g., Shafer et al. 2001, Buzacott 2002). The current result may indicate a benefit in being able to match a diverse set of worker capabilities with the wider range of demand requirements.

Table 6. Regression analysis of the unmet demand when DMC bounds are exceeded in relation to environmental variables

\begin{tabular}{|c|c|c|c|c|c|}
\hline Predictor & Coefficient & SE Coeff. & $t$ & $p$ & Regression statistics \\
\hline Constant & 116.17 & 2.03 & 57.12 & $<0.001$ & $R-S q=26.19 \%$ \\
\hline JTC & -117.36 & 4.41 & -26.63 & $<0.001$ & $R-S q(a d j)=26.15 \%$ \\
\hline \multicolumn{6}{|c|}{ With Demand-Mix Coverage } \\
\hline Predictor & Coefficient & SE Coeff. & $t$ & $p$ & Regression statistics \\
\hline Constant & 70.299 & 1.785 & 39.37 & $<0.001$ & $R-S q=43.2 \%$ \\
\hline JTC & -24.186 & 2.705 & -8.94 & $<0.001$ & $R-S q(\operatorname{adj})=43.2 \%$ \\
\hline DMC & -23.187 & 0.611 & 37.97 & $<0.001$ & \\
\hline
\end{tabular}


A sample of these relations is presented in Table 7, wherein the mean and standard deviations of levels of unmet demand when DMC bounds are exceeded, for various levels of $\mathrm{DMC}$, are presented. The data in the table corresponds to instances with DMC. We note that with fixed demand, FD,DMC bounds are not relevant by definition. We note that the highest levels of unmet demand correspond with the lowest DMC levels in the table. We can reason that, when a low demand-mix coverage conditions exist, workers' cross-training will be more limited and correspondingly, when DMC bounds are exceeded, the unmet demand will tend to be at higher levels.

Table 7. Unmet Demand When DMC Bounds Are Exceeded

\begin{tabular}{cccc|cccc}
\hline $\begin{array}{c}\text { DMC } \\
\text { level }\end{array}$ & $N$ & Mean & StDev & $\begin{array}{c}\text { Unmet Demand } \\
\text { level }\end{array}$ & $N$ & Mean & StDev \\
\hline 0.989 & 100 & 73.32 & 38.21 & 2.180 & 100 & 3.33 & 3.63 \\
1.084 & 100 & 41.85 & 23.05 & 2.229 & 100 & 2.40 & 2.96 \\
1.175 & 100 & 28.93 & 16.87 & 2.344 & 100 & 1.93 & 2.35 \\
1.743 & 100 & 6.94 & 5.64 & 2.355 & 100 & 3.53 & 3.86 \\
1.892 & 100 & 7.08 & 6.80 & 2.403 & 100 & 1.85 & 2.54 \\
2.048 & 100 & 4.67 & 8.85 & 2.608 & 100 & 1.00 & 1.02 \\
2.066 & 100 & 3.40 & 4.39 & 2.979 & 100 & 1.85 & 2.86 \\
2.066 & 100 & 8.47 & 11.20 & 3.098 & 100 & 1.24 & 3.64 \\
2.113 & 200 & 3.37 & 3.52 & 3.207 & 100 & 0.95 & 1.41 \\
2.153 & 100 & 5.27 & 7.40 & & & & \\
\hline
\end{tabular}

With respect to the absenteeism bound, in Table 8, the unmet demand is positively related to DMC. That is, absent workers and high demand-mix coverage, together lead to higher levels of unmet demand. We can posit that the higher cross-training necessary to cope with higher DMC will be also used to cover absences. The JTC results were not significant for unmet demand due to absences both with FD and with DMC. 


\section{Table 8. Regression analysis of unmet demand when exceeding AC bound in relation to environmental variables}

\begin{tabular}{|c|c|c|c|c|c|}
\hline Predictor & Coefficient & SE Coeff. & $T$ & $p$ & Regression statistics \\
\hline Constant & 37.40 & 1.20 & 31.17 & $<0.001$ & $R-S q=0.16 \%$ \\
\hline JTC & -4.69 & 2.60 & -1.80 & 1.000 & $R-S q($ adj $)=0.11 \%$ \\
\hline
\end{tabular}

With Demand-Mix Coverage

\begin{tabular}{|c|c|c|c|c|c|}
\hline Predictor & Coefficient & SE Coeff. & $T$ & $p$ & Regression statistics \\
\hline Constant & 29.501 & 1.020 & 28.93 & $<0.001$ & $R-S q=35.1 \%$ \\
\hline JTC & 4.329 & 1.545 & 2.80 & 0.005 & $R-S q(\operatorname{adj})=35.0 \%$ \\
\hline DMC & 11.4121 & 0.3489 & -32.71 & $<0.001$ & \\
\hline
\end{tabular}

\section{Conclusions}

In recent years the literature has made clear the need to balance costs and rewards from implementing cross-training and other work sharing systems. In this paper, we examined several cross-training policies, for managing the operations of teams of workers. The work environment is characterized by conditions with worker absenteeism, and demand-mix coverage. We conducted a range of experiments to consider the performance of these policies for meeting demand, and maintaining low costs. The results indicate that simply minimizing costs, while straightforward and effective, may not adequately address issues of robustness with respect to meeting demand given uncertain conditions, such as demand-mix coverage and absenteeism.

While alternate robustness characteristics will add to the current study, the current results indicate that a policy such as minimizing cross-training costs as a first priority, and then equalizing the cross-training level among the workforce, may provide an improvement in terms of robustness. Focusing solely on meeting demand will tend to increase cross-training costs, if both criteria are not considered in an overall policy. This is the main result obtained, as robustness against demand-mix variability and absenteeism can be improved, without any additional cost. From managerial point of view, this result provides and directly applicable criteria to the day to day decisions taking regarding cross-training. The effect of the environmental factors has also been analyzed. It is concluded that the greater the task similarity, 
the less costly is the necessary cross training, and the less demand goes unmet due to demandmix variation. Similarly, when cross-training is used to help to meet a level of demand-mix variation, there may be greater cross-training costs.

We note that this study investigated a specific set and range of scenarios, as well as performance metrics. It will be of considerable interest for future research to expand this set to include other relevant sources of uncertainty in addition to demand, and absenteeism. These may include, individual human differences, and non-stationary shifts in demand, and performance characteristics.

\section{References}

AGNIHOTHRI, S. R. \& MISHRA, A. K. 2004. Cross-training Decisions in Field Services with Three Job Types and Servers. Decision Sciences, 35, 239-257.

BOKHORST, J.A.C, AND SLOMP, J. 2007. Design and Operation of a Cross Trained Workforce, in Workforce Cross Training (ed. D. Nembhard). CRC-Taylor-and Francis.

BRUSCO, M. \& JOHNS, T. 1998. Staffing a multiskilled workforce with varying levels of productivity: an analysis of cross-training policies. Decision Sciences, 29, 499-516.

BUZACOTT, J. 2002. "The Impact of Worker Differences on Production System Output," International Journal of Production Economics, 78, 37-44.

CHAKRAVARTHY, S. R. \& AGNIHOTHRI, S. R. 2005. Optimal Workforce Mix in Service Systems with Two Types of Customers. Production \& Operations Management, 14, 218-231.

EASTON FF (2014) Service Completion Estimates for Cross-trained Workforce Schedules under Uncertain Attendance and Demand. Production and Operations Management 23, 660-675.

EASTON, F.F., 2011. "Cross-Training Performance in Flexible Labor Scheduling Environments," IIE Transactions, 43, 589-603.

FEI, X. and Mahmassani, H.S., 2011. "Structural Analysis of Near Optimal Sensor Locations for a Stochastic Large scale Network," Transportation Research Part C: Emerging Technologies, 19, 440-453. 
GIANNIKOS, I. 2010. "Fuzzy Goal Programming models for analyzing demand coverage," 18(1), 185-202. TOP Special Issue on EWGLA XVII (ed. M. Landete)

GNANLET A, GILLAND W (2014) Impact of Productivity on Optimal Cross-Training Decisions. European Journal of Operational Research, 238, 254-269.

GONG, J., WANG, L. \& ZHANG, S. 2011. A New Workforce Cross-Training Policy for a Ushaped Assembly Line Information and Management Engineering. In: ICCIC 2011, Part V, CCIS 235, 529-536, Springer-Verlag Berlin Heidelberg.

HOPP, W. J., TEKIN, E. \& OYEN, M. P. V. 2004. Benefits of Skill Chaining in Serial Production Lines with Cross-Trained Workers. Management Science, 50, 83-98.

JORDAN, W.C., INMAN, R. R., and BLUMENFELD, D. E., 2004. "Chained cross-training of workers for robust performance," IIE Transactions, 36, 953-967

MARENTETTE, K.A., JOHNSON, A.W., AND MILLS, L. 2009. "A Measure of Cross Training Benefits Versus Job Skill Specialization," Computers and Industrial Engineering, 57, 937-940. OLIVELLA, J. AND NEMBHARD, D. 2015. Calibrating Cross-Training to Meet Demand Mix Variation and Employee Absence. European Journal of Operational Research. In Press, Accepted Manuscript. Available online 22 July 2015.

SHAFER, S.M., D. A. NEMBHARD, M.V. UZUMERI, (2001) "The Effects of Worker Learning, Forgetting, and Heterogeneity on Assembly Line Productivity , "Management Science, 47, 1639-1653.

TEKIN, E., HOPP, W. J. \& VAN OYEN, M. P. 2002. Benefits of Skill Chaining in Production Lines with Cross-Trained Workers: An Extended Abstract, Manufacturing \& Service Operations Management, 4, 17-20.

WIROJANAGUD, P., GEL, E., Fowler, J.W., and Cardy, R. 2007. Modelling inherent worker differences for workforce planning. International Journal of Production Research, 45, 525-553. 